Educación Física y Ciencia, vol. 20, nº 4, e065, octubre-diciembre 2018. ISSN 2314-2561

Universidad Nacional de La Plata.

Facultad de Humanidades y Ciencias de la Educación.

Departamento de Educación Física

\title{
A gestão do evento Xterra Camp Paraná: Análise do seu impacto na perspectiva dos participantes
}

The management of the Xterra Camp Paraná event: Analysis of its impact from the participants' perspective

\author{
Gustavo Bavaresco \\ Faculdade de Desporto da Universidade do Porto, Brasil \\ gustavobava@gmail.com
}

\section{Resumo:}

O estudo pretende ser um contributo para os gestores de eventos esportivos, a partir das perspectivas dos participantes no evento XTerra Camp Paraná que decorreu fora dos grandes centros urbanos brasileiros. Trata-se de um estudo de natureza quantitativa descritiva, no qual se utilizou um questionário, revisado por professores universitários e gestores. Os principais resultados evidenciam (i) um perfil de participantes de maioria de sexo masculino e não residentes; (ii) valores de gastos entre 100 a 500 reais; e (iii) elevada satisfação com o percurso, organização do evento e região. Conclui-se que, na ótica dos participantes, o evento globalmente lhes agradou por ter tido uma organização eficiente e por terem vivenciado experiências na região ao nível turístico.

PALAVRAS-CHAVE: Esportes, Natureza, Organização e administração, Turismo.

\section{Abstract:}

The study intends to be a contribution to the managers of sporting events, from the perspective of the participants in the event XTerra Camp Paraná that took place outside the large Brazilian urban centers. This is a quantitative descriptive study, in which a questionnaire was used, reviewed by specialists such as university professors and managers. The main results show (i) a profile of male and non-resident majority participants; (ii) expenses ranged between 100 and 500 reais; and (iii) high satisfaction with the course, organization of the event and region. It is concluded that, from the perspective of the participants, they were globally satisfied with the event because of its efficient organization and the tourist activities associated to it.

KEYWORDS: Sports, Nature, Organization and administration, Turism.

\section{INTRODUÇÃO}

Na última década é notório o crescimento do número e da importância dos eventos esportivos, bem como do impacto produzido na sociedade e a sua repercussão. $\mathrm{O}$ crescimento e a proliferação de eventos nas sociedades contemporâneas são fenomenais. De comunidades pequenas a nações inteiras, os eventos são usados como ferramentas para uma variedade de propósitos. Embora os eventos sejam muito diversificados quanto a sua natureza, os motivos que estão na base do seu planeamento prendem-se com o determinismo político ou econômico (Ziakas \& Costa, 2011).

Os eventos esportivos são considerados catalizadores do crescimento econômico e turístico nos locais que se realizam. Porém, estes fatores têm sido investigados em megaeventos esportivos e pouca atenção se tem dado aos eventos de pequena e média dimensão (Agha \& Taks, 2015; A. Barajas, Coates, \& SanchezFernandez, 2016). No entanto, (Coates, 2012; Lima, Maia, Lobosco, \& Moraes, 2016) sugerem que os eventos esportivos em pequena escala podem ter efeitos positivos para as comunidades anfitriãs.

Para elucidar, a literatura em eventos esportivos de pequena escala é dispersa (Gibson, Willming, \& Holdnak, 2003; Lee, Harris, \& Lyberger, 2010), com o maior foco em estudos de impacto de larga escala ou megaeventos. O leque de estudos em eventos de pequena escala é uma consequência de dificuldades em executar tais estudos como também à medida em que o tamanho do evento diminui (Matheson, 2006; Mondello \& Rishe, 2004).

O grau de dificuldade em realizar estudos em eventos em espaços abertos, como por exemplo, ralis e ciclismo de estrada, pode estar relacionado com a aplicação dos questionários, a qual é mais facilitada em 
espaços fechados (Desbordes, 2007). Todavia, existem alguns estudos em rali (Hassan \& Connor, 2009), ciclismo (Collins, Munday, \& Roberts, 2012) ou maratonas (Barquet, Brida, Osti, \& Schubert, 2011; Wicker, Hallmann, \& Zhang, 2012).

Para além dos espaços onde se realizam os eventos, existem outros critérios que diferenciadores para a sua tipologia. Barajas, Salgado, and Sánchez (2012), procederam a uma ampliação da tipologia proposta por (Gratton, Dobson, \& Shibli, 2000) que tinha estabelecido os Tipos A, B, C, e D e da tipologia expandida por (Wilson, 2006) ao ter criado o Tipo E. Desta maneira, Barajas et al. (2012), propõe que os eventos desportivos podem ser classificados em sete tipos: Tipo A Tipo B, Tipo C1, Tipo C2, Tipo D1, Tipo D2 e Tipo E, sendo que, sucintamente, são diferenciações relacionadas com características gerais (entre outras, a regularidade, $\mathrm{o}$ midiatismo, a assistência de espectadores) e econômicas (entre outras, a frequência, competitividade entre países ou cidades para a candidatura, magnitude da atividade econômica).

Efetivamente, a caracterização dos eventos esportivos ajuda na seleção de metodologias para estudar os impactos (Li \& Jago, 2013). Fornece igualmente informações relativamente a critérios que gerem aspectos positivos e qual o foco para ter mais impacto, assim como relativamente a aspectos que não são tão benéficos, mas que podem melhorar.

A gestão de eventos e\# uma das atividades que todos os profissionais da área do esporte desenvolvem ao menos uma vez em sua carreira ou com certa regularidade. Seja na escola, na empresa ou no município sendo uma das expressões mais característica do esporte, obviamente dimensionados a\# realidade e objetivos previamente definidos (Sarmento, Pinto, Costa, \& Silva, 2011).

Os autores Barajas et al. (2012) afirmam que a validade dos resultados do impacto econômico depende de uma variedade de questões metodológicas, incluindo o tipo de competição, alcance geográfico, duração, participantes, espectadores, localização, necessidades de infraestrutura e cronograma.

Este estudo tem como objeto de investigação um evento com características especiais, trata-se do “XTERRA Camp Paraná 2016” e decorreu em uma área não densamente povoada, mais especificamente na região rural da cidade de Ponta Grossa - Paraná. Este evento esportivo insere-se no quadro do circuito XTERRA Brasil que como é sabido diz respeito a um festival multi-esportivo que possui provas de Triatlhon off-road, Night Run, Trail Run, Mountain Bike CUP, Mountain Bike Enduro, Endurance, Swim Challenge e Kids Mini-corrida que decorrem anualmente em regiões em contato com a natureza.

Existem duas principais razões para a análise deste evento esportivo. Sendo a primeira vez que ocorre na região sul do país dada a sua importância sócio esportiva, esta marca (XTERRA) já ocorreu etapas nas demais regiões do Brasil e regularmente tem sido realizado a mais de 10 anos com diversas etapas durante o ano, merece ser alvo de investigação. Em segundo lugar, enquanto é um evento de pequena a média escala, poderá ajudar a extrair pontos positivos aos organizadores de eventos esportivos desta magnitude.

Por esses motivos, esta investigação pretende oferecer conhecimentos úteis a esse público como objetivo do presente trabalho desenhar a experiência deste evento. Em que possa contribuir para melhorar o trabalho gestionário de organizadores de eventos esportivos e cidades anfitriãs. Para projetar eventos com essas características e conhecer os elementos-chave sobre os quais agir para aumentar o impacto positivo e minimizando o negativo.

Portanto, nosso estudo consiste não apenas na apresentação dos principais resultados mas apresentar a experiência organizacional de um evento que decorreu pela primeira vez na região sul do Brasil o XTERRA Camp Paraná no ano de 2016. Como os conhecimentos gerados poderão auxiliar no refinamento de pesquisas sobre médios e pequenos eventos, propiciando lições mais consistentes para futuras iniciativas, eventualmente, esse tipo de pesquisa poderia ser aproveitado como ferramenta de análise e decisão por organizadores e decisores políticos na realização de mais eventos, talvez menores, como já recomenda repetitivamente a literatura. 


\section{Metodologia}

O presente estudo caracteriza-se por ser de natureza quantitativo, descritivo. Trata-se de um estudo de caso, uma vez que envolve a apresentação e a análise de informações detalhadas de um ou mais assuntos em relação a um evento (Edwards \& Skinner, 2009; Veal \& Darcy, 2014; Yin, 2013).

De acordo com (Barajas et al., 2012; Gratton et al., 2000; Wilson, 2006) podemos classificar o evento estudado, como uma classificação Tipo $\mathrm{C} 1$, consistindo em ser irregular ou regular que trocam de sede, maior frequência de celebração, assistência de espectadores e competidores internacionais, atividade econômica limitada e incerto término de impacto econômico e acrescentamos a existência do interesse midiático.

A recolha de informação foi realizada em dois dias seguidos por meio da aplicação de um questionário físico $a d-h o c$, foi submetido a revisão por um grupo de experts, composto por 4 professores universitários especializados em gestão esportiva e pela própria direção do evento, com mais de 10 anos de experiência no desenvolvimento deste tipo de eventos, a uma amostragem aleatória simples com a margem de erro fixada a um 5\% (Gil, 2010; Marotti et al., 2008) aos participantes do evento XTERRA Camp Paraná nos dias 15 e 16 de outubro de 2016, realizado na região rural da cidade de Ponta Grossa, Paraná - Brasil. Esta pesquisa de campo foi realizada por três pesquisadores pelo meio da abordagem pessoal direta.

Neste sentido foram aplicados 170 questionários, que após a tabulação e limpeza dos dados resultaram no total 125 respostas consideradas válidas, dado que se descartaram questionários incompletos ou mal preenchidos. Importa referir que o questionário aplicado integrava as seguintes dimensões de análise: i) dados sociodemográficos, ii) participantes e gastos e; iii) satisfação com o evento. Para as respostas ao questionário foi utilizada a escala Likert de 5 pontos.

$\mathrm{Na}$ dimensão de dados sociodemográficos foram analisadas as variáveis de sexo e idade. Em relação à dimensão dos participantes e gastos foram analisadas as variáveis de participantes, hospedagem, tempo de estadia, meio de transporte e dinheiro gasto durante o período do XTERRA Camp Paraná. Na dimensão satisfação com o evento as variáveis percurso da corrida, organização do evento, gastronomia, vida cultural da região, monumentos e ócio da região foram avaliadas.

Com o objetivo de proceder à organização e limpeza dos dados recolhidos, procedemos à sua tabulação e processamento, tendo sido criado uma base de dados em documento, utilizando-se o software Microsoft Office Excel 2016.

Para a análise e tratamento dos dados recorremos ao pacote estatístico $S P S S^{\bullet}$ versão 23 e foram analisados a partir da estatística descritiva (média e frequência relativa).

\section{Resultados E Discuss Ão}

\section{I) Dados Sociodemográficos}

O festival multi-esportivo além de congregar 784 atletas para competir em 08 provas disponibilizadas (Triathon off-road, Trail Run, Short Night Run, Mountain Bike Cup Pro, Mountain Bike Cup Sport, Marathon, Swim Challenge Kids Mini-corrida), reuniu cerca de 4000 visitantes em dois dias de competições.

As 125 respostas consideradas válidas, foram distribuídas entre 87 (69,6\%) do sexo masculino e 38 (30,4\%) do sexo feminino. A idade encontra-se de 16 aos 69 anos, sendo a média de idade de 34.43 anos (SD = 13.82). Os resultados encontrados sobre os participantes do XTERRA Camp Paraná, apresentaram características idênticas a diversos estudos realizados em diferentes contextos e sugerem que o participante deste tipo de evento é em sua maioria do sexo masculino, com a idade entre adultos-jovens (Hassan \& Connor, 2009; Kaplanidou \& Vogt, 2007; Wicker et al., 2012). 
Podemos observar na Tabela 1 que a maior concentração de atletas está no Triatlhon Off-Road totalizando $(27,2 \%)$ e os demais estão distribuídos nas outras modalidades, conforme discriminado.

Tabela 1. Divisão de participantes por modalidade

\begin{tabular}{lcccc}
\hline \multirow{2}{*}{ Modalidade } & \multicolumn{2}{c}{ Masculino } & \multicolumn{2}{c}{ Feminino } \\
\cline { 2 - 5 } & f.r & $\%$ & f.r & $\%$ \\
\hline Mountain Bike Cup Pro & 14 & 11,20 & 5 & 4 \\
Mountain Bike Cup Sport & 16 & 12,80 & 3 & 2,40 \\
Triathon Off-Road & 24 & 19,20 & 10 & 8 \\
Swim Challenge & 4 & 3,20 & 2 & 1,60 \\
Short Night Run & 12 & 9,60 & 2 & 1,60 \\
Trail Run & 13 & 10,40 & 14 & 11,20 \\
Marathon & 4 & 3,20 & 2 & 1,60 \\
\hline
\end{tabular}

Fonte: Elaborado pelos Autores (2018).

\section{II) Participantes e Gastos}

Os resultados apresentam, as variáveis de participantes, hospedagem, tempo de estadia, meio de transporte e dinheiro gasto durante o período do XTERRA Camp Paraná.

Os resultados sugerem dois tipos de participantes, os não residentes que são no total 105 atletas $(84 \%)$ e os residentes 20 atletas (16\%). Assim, em relação aos participantes não residentes que pernoitaram 67 (63,8\%), observamos que se hospedaram em hotéis, pousadas e outros locais, sendo a estadia de até 3 dias $(62,7 \%)$ a maioritária.

O resultado relacionado ao "tempo de estadia" entra em acordo com o que foi apontado por Daniels and Norman (2003) onde os participantes passaram em média dois dias na cidade do evento.

Tabela 2. Gastos participantes não residentes e residentes

\begin{tabular}{|c|c|c|c|c|}
\hline \multirow[t]{2}{*}{ Participantes } & \multicolumn{2}{|c|}{ Não Residentes } & \multicolumn{2}{|c|}{ Residentes } \\
\hline & f.r & $\%$ & f.r & $\%$ \\
\hline Pernoitaram & 67 & 63,8 & 20 & 16 \\
\hline Não pemoitaram & 38 & 36,2 & - & - \\
\hline \multicolumn{5}{|l|}{ Hospedagem } \\
\hline Hotéis & 30 & 44,77 & & \\
\hline Pousadas & 13 & 19,4 & & \\
\hline Outros locais & 24 & 35,8 & & \\
\hline \multicolumn{5}{|l|}{ Estadia } \\
\hline Até 3 dias & 42 & 62,7 & & \\
\hline 3 a 5 dias & 18 & 26,86 & & \\
\hline 5 a 10 dias & 7 & 10,44 & & \\
\hline \multicolumn{5}{|l|}{ Meio de Transporte } \\
\hline Carro & 47 & 37,6 & 11 & 8,8 \\
\hline Avião & 10 & 8 & 0 & 0 \\
\hline Ônibus & 22 & 17,6 & 4 & 3,2 \\
\hline Outro tipo de transp. & 26 & 20,8 & 5 & 4 \\
\hline \multicolumn{5}{|l|}{ Gastos } \\
\hline 50 a 100 reais & 9 & 7,2 & 16 & 12,8 \\
\hline 100 a 500 reais & 45 & 36 & 39 & 31,2 \\
\hline 500 a 1000 reais & 14 & 11,2 & 2 & 1,6 \\
\hline Acima de 1000 reais & 0 & 0 & 0 & 0 \\
\hline
\end{tabular}

Fonte: Elaborado pelos Autores (2018). 
O meio de transporte utilizado de acordo com a amostra total de nossa pesquisa apresenta que entre os participantes residentes e não residentes o carro é o mais utilizado. Observa-se que, como a cidade anfitriã não possuía aeroporto de grande porte, os atletas que vieram de avião, após chegarem a Curitiba, capital do Estado do Paraná com aeroporto, utilizaram o meio de transporte do ônibus, carro e outro tipo de transporte, para se deslocarem para a cidade anfitriã e para o local do evento. O estudo de Hayek (2014) que evidencia que as corridas de rua estão em constante crescimento, recomenda que os organizadores desses eventos devem ter um planejamento para a gestão de trânsito, principalmente em grandes cidades. Desta forma, os participantes, moradores e usuários destes locais não ficarão tão prejudicados e terão mais garantias para a sua segurança e para a realização do evento. Acrescente-se que, inclusive, para eventos em regióes afastadas da cidade, tal planejamento para a gestão de trânsito é mesmo decisivo.

Em relação aos custos, foi perguntado quanto gastou ou pretende gastar durante o evento e obtivemos que 84 atletas $(67,2 \%)$, residentes e não residentes, gastaram durante o evento entre 100 a 500 reais. Nestes valores estão embutidos deslocamentos, hospedagem, restaurantes, cafés, entre outros serviços consumidos na cidade acolhedora do evento e no próprio evento. Resultados de Jiménez-Naranjo, Coca-Pérez, GutiérrezFernández, and Sánchez-Escobedo (2016) para um evento realizado em Espanha, revelaram que um participante gastou uma média de 109,50 euros. Contudo, as despesas do participante variam, dependendo se ele é residente ou não. Assim, a despesa média de um participante residente ou não residente que não permanece à noite é bastante semelhante e gira em torno de 55 Euros. No entanto, quando analisada a despesa dos participantes não residentes que pernoitam na cidade, eles podem provar que o valor aumenta mais de $300 \%$, gerando o montante de 188,39 euros. Por tudo isso, concordamos com Barajas et al. (2016) ao afirmarem que o significado da validade dos resultados do impacto econômico depende de uma variedade de questões metodológicas.

\section{III) Satisfação com o Evento}

Na dimensão satisfação com o evento as variáveis percurso das modalidades esportivas, organização do evento, gastronomia, vida cultural da região, natureza e ócio da região foram avaliadas.

O percurso das modalidades esportivas foi avaliado positivamente por 85,6\% de participantes. Quanto à hidratação durante as competições a sua avaliação foi entre o bom e muito bom, totalizando (68\%) dos participantes. Em relação ao fornecimento de frutas e outros alimentos observamos que os valores são semelhantes entre o Ruim e Muito Bom, pelo que os organizadores devem tomar cuidado com este quesito para que se minimizem os pontos negativos.

Tabela 3. Satisfação quanto ao percurso das modalidades

\begin{tabular}{lcccccccccc}
\hline & \multicolumn{3}{c}{$\begin{array}{c}\text { Muito } \\
\text { Ruim }\end{array}$} & \multicolumn{2}{c}{ Ruim } & \multicolumn{2}{c}{ Regular } & \multicolumn{2}{c}{ Bom } & \multicolumn{2}{c}{ Muito Bom } \\
\cline { 2 - 11 } & f.r & $\%$ & f.r & $\%$ & f.r & $\%$ & f.r & $\%$ & f.r & $\%$ \\
\cline { 2 - 11 } & - & - & 14 & 11,2 & 26 & 20,8 & 45 & 36 & 40 & 32 \\
Hidratação & - & - & 4 & 3,2 & 14 & 11,2 & 45 & 36 & 62 & 49,6 \\
$\begin{array}{l}\text { Marcação de percurso } \\
\begin{array}{l}\text { Frutas e outros alimentos } \\
\text { oferecidos }\end{array}\end{array}$ & 12 & 9,6 & 20 & 16 & 33 & 26,4 & 26 & 20,8 & 34 & 27,2 \\
\hline
\end{tabular}

Fonte: Elaborado pelos Autores (2018).

Quanto a satisfação para com a organização do evento, foram observados: o processo de inscrição, o "Kit atleta”, o Acesso ao evento, o Seminário técnico aos atletas, a Programação, a Estrutura e organização da arena, o Atendimento durante o festival e a Praça de alimentação, conforme melhor ilustrado na Tabela 4. 
Tabela 4. Satisfação para com a organização do evento

\begin{tabular}{|c|c|c|c|c|c|c|c|c|c|c|}
\hline & \multicolumn{2}{|c|}{$\begin{array}{l}\text { Muito } \\
\text { ruim }\end{array}$} & \multicolumn{2}{|c|}{ Ruim } & \multicolumn{2}{|c|}{ Regular } & \multicolumn{2}{|c|}{ Bom } & \multicolumn{2}{|c|}{ Muito bom } \\
\hline & f.r & $\%$ & f.r & $\%$ & f.r. & $\%$ & f.r & $\%$ & f.r & $\%$ \\
\hline Processo de inscrição & 5 & 4 & 14 & 11,2 & 30 & 24 & 28 & 22,4 & 48 & 38,4 \\
\hline Kit atleta & - & - & 9 & 7,2 & 61 & 48,8 & 34 & 27,2 & 21 & 16,8 \\
\hline Acesso ao evento & 4 & 3,2 & 13 & 10,4 & 21 & 16,8 & 31 & 24,8 & 56 & 44,8 \\
\hline Seminário técnico aos atletas* & - & - & - & - & 22 & 51,17 & 10 & 23,25 & 11 & 25,58 \\
\hline Programação & 2 & 1,6 & 5 & 4 & 16 & 12,8 & 22 & 17,6 & 35 & 28 \\
\hline Estrutura e organização da arena & 12 & 9,6 & 13 & 10,4 & 45 & 36 & 35 & 28 & 20 & 16 \\
\hline Atendimento antes e durante o evento & 5 & 4 & 5 & 4 & 31 & 24,8 & 26 & 20,8 & 58 & 46,4 \\
\hline Praça de alimentação & 6 & 4,8 & 8 & 6,4 & 26 & 20,8 & 45 & 36 & 40 & 32 \\
\hline
\end{tabular}

* 82 atletas não responderam esta questão Fonte: Elaborado pelos Autores (2018).

Para o processo de inscrição observamos que cerca de $15 \%$ dos atletas não gostaram, contudo, a satisfação foi predominante com $60 \%$ de respostas. Um processo de inscrição eficiente é fundamental para causar uma boa primeira impressão do evento (Gillespie \& McDonald, 2015, p. 232).

No que tange ao "Kit atleta", 48,8\% dos participantes investigados identificaram-no como regular. Esta percentagem pode indiciar aos organizadores que terão de fazer algo mais para que, também neste quesito, o evento seja memorável.

Chegar ao evento em boas condições de acesso, no que respeita ao tipo de estradas, sinalização e estacionamento, é algo que contribui bastante para a satisfação geral. Sendo a realização das várias competições em regiões não densamente povoadas, sobretudo em regiões rurais, pode, eventualmente, não existir sinal de telefone, internet e GPS (Global Positioning System). Assim, as estradas que por vezes não são pavimentadas como nas cidades, devem estar bem sinalizadas e com marcações para indicar o caminho e disponibilizar o trajeto a partir de um mapa pré-evento. Tais circunstâncias foram devidamente atendidas pelos organizadores do evento pois, aproximadamente, $70 \%$ dos participantes assinalaram ter tido um notável acesso ao mesmo.

No que respeita ao "Seminário técnico" presume-se que houve pouca adesão ao mesmo pois 82 dos questionados não o frequentaram. Contudo, os que assistiram ao seminário técnico, 11 (25,58\%) avaliaramno como muito bom, $10(23,25 \%)$ como bom e $22(51,17 \%)$ como regular.

Quanto à "Programação", tendo o evento decorrido em três dias consecutivos, sendo um somente para a entrega do "kit atleta", os participantes responderam favoravelmente: 35 participantes (28\%) referiram muito bom e 22 (17,6\%) bom. De acordo com Giacomelli and Henkelman (2012) os organizadores devem tomar conhecimento das datas para que os eventos encontrem uma forma de atrair os participantes e entusiastas, bem como estar atento aos horários e as questões climáticas.

No que diz respeito à "Estrutura e organização da arena", os organizadores devem também deter grandes precauções. Como já referido, por ser em região não densamente povoada, isto é, numa região rural, é difícil encontrar uma estrutura pronta, em bom estado, disponível e adequada para eventos deste género e que comporte uma grande quantidade de pessoas. Tal determina que os organizadores construam ou aluguem, espaços e equipamentos para a realização do evento. Seguindo as exposições de Giacomelli and Henkelman (2012) a elaboração de contratos é estimulada para a prevenção de qualquer má interpretação das funções entre as partes, a fim de evitar problemas entre organizadores e colaboradores. É necessário criar um vinculo de confiança entre os contratantes.

Além do sistema de segurança, os organizadores devem ser cuidadosos em relação aos tipos de espaços disponíveis que eventos deste género e magnitude necessitam, entre outros, para os patrocinadores, apoiantes, premiação, praça de alimentação, primeiros atendimentos, estacionamento, entrega de kits, e local para 
higiene pessoal (Gillespie \& McDonald, 2015, p. 227). Os participantes colocam como regular 36\% e bom $28 \%$ a estrutura e organização da arena do evento. Porém, não podemos deixar de registar que foi o ponto que obteve maiores respostas de visão negativa $20 \%$, como tal os organizadores deste evento devem assegurar maiores cuidados e precauções neste âmbito.

Reportando ao "Atendimento antes e durante o evento" 46,4\% dos participantes colocam como muito bom; $20,8 \%$ como bom; $24,8 \%$ como regular e $4 \%$ como ruim e muito ruim. Tal expressão por parte, tanto dos participantes como dos espectadores, é reveladora da satisfação neste quesito. Para além de outros aspectos, o público possivelmente recomendaria o evento pela forma como foram atendidos. Investigações de (Gillespie \& McDonald, 2015, p. 235); Kerwin, Warner, Walker, and Stevens (2015) e Oliveira and Costa (2016) demonstram que os voluntários são o ponto estruturante para o caminhar de um excelente evento e os primeiros a terem contato com participantes e espectadores. Neste sentido, devem ter formação para se relacionarem com os intervenientes auxiliando-os ao máximo e com entusiasmo na execução das diversas atividades. Se assim for, são reconhecidos pelo seu trabalho e atrairão o retorno daqueles para próximos eventos.

Por último, mas não sendo de menor importância abordamos a "Praça de alimentação", dizendo que em eventos como o estudado, com uma duração maior do que o comum, é fundamental que haja um local específico para participantes e expectadores se alimentarem. Na verdade, ocorrem muitas modalidades durante todo o dia e como as pessoas disfrutam do seu tempo de lazer, com a família e amigos, no contato com a natureza, torna-se propício o consumo de refeições e bebidas. Efetivamente, neste domínio os organizadores do XTERRA Camp Paraná também estiveram bem, como é demonstrado por $68 \%$ das respostas positivas dos atletas.

Para a satisfação da região quanto à vida cultural, ócio e natureza, foram observados os seguintes itens: escolha do local, a importância do evento para a cidade e se visitou ou irá visitar atrativos turísticos da região, conforme demonstrado na Tabela 5.

Tabela 5. Satisfação da região quanto a vida cultural, ócio e natureza

\begin{tabular}{|c|c|c|c|c|c|c|c|c|c|c|}
\hline \multirow{4}{*}{$\begin{array}{l}\text { Escolha do local do evento } \\
\text { O evento para a cidade }\end{array}$} & \multicolumn{2}{|c|}{ Muito ruim } & \multicolumn{2}{|c|}{ Ruim } & \multicolumn{2}{|c|}{ Regular } & \multicolumn{2}{|c|}{ Bom } & \multicolumn{2}{|c|}{ Muito bom } \\
\hline & f.r & $\%$ & f.r & $\%$ & f.r & $\%$ & f.r & $\%$ & f.r & $\%$ \\
\hline & 7 & 5,6 & 10 & 8 & 37 & 29,6 & 47 & 37,6 & 24 & 19,2 \\
\hline & 3 & 2,4 & 14 & 11,2 & 38 & 30,4 & 27 & 21,6 & 43 & 34,4 \\
\hline \multirow{3}{*}{$\begin{array}{l}\text { Visitou ou irá visitar atrativos } \\
\text { turísticos da região }\end{array}$} & \multicolumn{2}{|c|}{ Não } & \multicolumn{2}{|c|}{ Talvez } & \multicolumn{2}{|c|}{ Sim } & & & & \\
\hline & f.r & $\%$ & f.r & $\%$ & f.r & $\%$ & & & & \\
\hline & 31 & 24,8 & 16 & 12,8 & 78 & 62,4 & & & & \\
\hline \multirow[t]{3}{*}{ Trouxe família e amigos } & \multicolumn{2}{|c|}{ Não } & \multicolumn{2}{|c|}{ Sim } & & & & & & \\
\hline & f.r & $\%$ & f.r & $\%$ & & & & & & \\
\hline & 34 & 27,2 & 91 & 72,8 & & & & & & \\
\hline
\end{tabular}

Fonte: Elaborado pelos Autores (2018).

Este assunto é de extrema importância para os organizadores e decisores políticos que laborem com o turismo e esporte. Saber se visitou, tem a intenção de visitar ou retornar à cidade onde decorre o evento, para percorrer atrativos da região e se trouxe família e amigos, são aspectos possíveis de mover a economia da região e cidade anfitriã do evento (Fedrizzi, Mendes, \& Schliemann, 2017; Kaplanidou \& Vogt, 2007; Shonk \& Chelladurai, 2008; Shonk, Greenwell, Bravo, \& Won, 2012; Wicker et al., 2012).

A escolha do local do evento foi registrada entre uma boa escolha e uma escolha regular. Já a importância do evento para a cidade apresenta uma maior diferença entre regular e muito bom. Resultados que podem ser compreensíveis pelo fato do evento ter sido realizado pela primeira vez na região sul do Brasil e ser um evento de pequena a média escala. Evidências que podem suscitar dúvidas nas pessoas quanto aos benefícios que pode gerar. Muitos estudos tratam dos megaeventos esportivos com impactos positivos e negativos para 
a cidade (Agha \& Taks, 2015; Barajas et al., 2016), mas ainda são incipientes e dispersas as pesquisas que abordam os impactos em eventos de menor dimensão (Gibson et al., 2003; Lee et al., 2010). Contudo, dos que existem, parece ser evidente que os eventos de menor dimensão trazem mais benefícios para as cidades e regiões (Coates, 2012; Lima et al., 2016).

É importante destacar que $62,4 \%$ dos participantes dizem que visitaram ou irão visitar atrativos turísticos da região. De acordo com Shonk and Chelladurai (2008) existem características específicas para um determinado destino, tais como o clima, a falta de opções alternativas, atrações ou escolhas de turismo esportivo, os custos de viagem. As questões relativas à segurança pessoal também podem influenciar a decisão quanto à intenção de retornar. Esta intenção pode ser focada no local do evento, no próprio evento, em ambos e acrescentamos as regióes turísticas e a própria cidade neste contexto.

A partir de resultados encontrados por Kaplanidou and Vogt (2007) a imagem de destino e a experiência passada com o destino influenciam significativamente as intenções de revisitar a região ou cidade com atividades de turismo esportivo. A satisfação com o evento e a imagem do evento foram importantes para a intenção de visitar a cidade e a maratona no estudo de (Wicker et al., 2012).

Observamos que muitos dos atletas $84 \%$ são participantes não residentes, donde se pode afirmar que se deslocam novas pessoas para a região e cidade. Não podemos expor quantas pessoas os não residentes trouxeram, mas observamos que $72,8 \%$ dos participantes dizem ter levado a família e amigos.

Por toda a experiência com este evento e os resultados alcançados, podemos referir que a gestão do esporte, designadamente na indústria de eventos esportivos assume uma posição estratégica dentro das políticas públicas que visam fomentar as atividades econômicas, gerar mais empregos para suas comunidades, mesmo que temporários, e consequentemente arrecadar impostos para a região e cidade anfitriá.

\section{Considerações Finais}

Com a presente investigação fica evidente que a organização de eventos de média e pequena escala, como o da presente investigação, é complexa, mas atrativa por envolver muitas variantes e centenas de intervenientes. Neste sentido, observa-se que os participantes tiveram uma ótima experiência com este evento, muitos deles tiveram a oportunidade de conhecer mais pontos turísticos da região. A organização foi muito eficiente, mas tem que cuidar em alguns aspectos para minimizar os pontos negativos e maximizar os positivos.

Pela quantidade de participantes no evento em estudo e atendendo às suas perspectivas o evento foi benéfico para a região, pois a maioria pernoitou longe de casa, com repercussóes benéficas para a economia e turismo na a cidade anfitriã. Porém, como qualquer outro, apresentou algumas dificuldades que devem ser consideradas pelos organizadores e decisores políticos.

Os resultados desta pesquisa abrem espaço para futuros estudos que façam comparações com outros eventos de mesmo gênero e também que investiguem as diferentes etapas do Xterra Brasil.

\section{REFERÊNCIAS}

Agha, N., \& Taks, M. (2015). A theoretical comparison of the economic impact of large and small events. International Journal of Sport Finance, 10, 103-121.

Barajas, A., Coates, D., \& Sanchez-Fernandez, P. (2016). Beyond retrospective assessment. Sport event economic impact studies as a management tool for informing event organization. European Research on Management and Business Economics, 22(3), 124-130.

Barajas, Á., Salgado, J., \& Sánchez, P. (2012). Problemática de los estudios de impacto económico de eventos deportivos. Estudios de economía aplicada, 30(2), 441-461.

Barquet, A., Brida, J. G., Osti, L., \& Schubert, S. (2011). An analysis of tourists' expenditure on winter sports events through the tobit censorate model. Tourism Economics, 17(6), 1197-1217. doi:10.5367/te.2011.0084 
Coates, D. (2012). Not-So-Mega Events. In W. Maennig \& A. Zimbalist (Eds.), International Handbook on the Economics of Mega Sporting Events (pp. 401). Cheltenham, UK: Edward Elgar Publishing.

Collins, A., Munday, M., \& Roberts, A. (2012). Environmental consequences of tourism consumption at major events: An analysis of the UK stages of the 2007 Tour de France. Journal of Travel Research, 51(5), 577-590.

Daniels, M. J., \& Norman, W. C. (2003). Estimating the economic impacts of seven regular sport tourism events. Journal of Sport \& Tourism, 8(4), 214-222.

Desbordes, M. (2007). A review of the economic impact studies done on the Tour de France: Methodological aspects and first results. International Journal of Sport Management and Marketing, 2(5-6), 526-540. doi:10.1504/ IJSMM.2007.013965

Edwards, A., \& Skinner, J. (2009). Qualitative research in sport management. Oxford: Elsevier Ltd.

Fedrizzi, V. L. P., Mendes, B. d. C., \& Schliemann, M. (2017). Tourism of events in Campos do Jordão: CJCVB strategies to attract events. Revista de Turismo Contemporâneo, 5(Edição Especial), 55-70.

Giacomelli, L. F., \& Henkelman, A. (2012). Organização de mega eventos esportivos: Cases e modelos. Revista Intercontinental de Gestão Desportiva, 2(2), 86-107.

Gibson, H. J., Willming, C. \& Holdnak, A. (2003). Small-scale event sport tourism: Fans as tourists. Tourism Management, 24(2), 181-190.

Gil, A. C. (2010). Como elaborar projetos de pesquisa. São Paulo: Atlas.

Gillespie, W. S., \& McDonald, M. (2015). Gestão de eventos esportivos. En P. d. S. Vance, V. M. J. Nassif, \& L. P. Masteralexis (Eds.), Gestao do esporte: Casos brasileiros e internacionais (pp. 217-240). Rio de Janeiro: LTC.

Gratton, C., Dobson, N., \& Shibli, S. (2000). The economic importance of major sports events: A case-study of six events. Managing Leisure, 5(1), 17-28.

Hassan, D. \& Connor, S. O. (2009). The socio-economic impact of the FIA World Rally Championship 2007. Sport in Society, 12(6), 709-724.

Hayek, F. B. A. (2014). Gestão do Trânsito para Atividades Esportivas na Via - Corridas de Rua na cidade de São Paulo. PODIUM Sport, Leisure and Tourism Review, 3(3), 45-56.

Jiménez-Naranjo, H. V., Coca-Pérez, J. L., Gutiérrez-Fernández, M. \& Sánchez-Escobedo, M. C. (2016). Cost-benefit analysis of sport events: The case of World Paddle Tour. European Research on Management and Business Economics, 22(3), 131-138.

Kaplanidou, K. \& Vogt, C. (2007). The Interrelationship between Sport Event and Destination Image and Sport Tourists' Behaviours. Journal of Sport \& Tourism, 12(3-4), 183-206.

Kerwin, S., Warner, S., Walker, M. \& Stevens, J. (2015). Exploring sense of community among small-scale sport event volunteers. European Sport Management Quarterly, 15(1), 77-92.

Lee, S., Harris, J. \& Lyberger, M. (2010). The economic impact of college sporting events: A case study of division IA football games. Event Management, 14(2), 157-165.

Li, S. \& Jago, L. (2013). Evaluating economic impacts of major sports events - a meta analysis of the key trends. Current Issues in Tourism, 16(6), 591-611.

Lima, E., Maia, T. S. T., Lobosco, A. \& Moraes, M. B. d. (2016). Megaeventos esportivos frente a pequenos eventos: Reflexões considerando-se a realidade brasileira recente. PODIUM Sport, Leisure and Tourism Review, 5(3), 89-110.

Marotti, J., Galhardo, A. P. M., Furuyama, R. J., Pigozzo, M. N., Campos, T. N. d., \& Laganá, D. C. (2008). Amostragem em pesquisa clínica: Tamanho da amostra. Revista de Odontologia da Universidade Cidade de São Paulo, 20(2), 186-194.

Matheson, V. A. (2006). Is smaller better? A comment on "comparative economic impact analyses" by Michael Mondello and Patrick Rishe. Economic Development Quarterly, 20(2), 192-195.

Mondello, M. J. \& Rishe, P. (2004). Comparative economic impact analyses: Differences across cities, events, and demographics. Economic Development Quarterly, 18(4), 331-342. 
Oliveira, L. B. d. \& Costa, F. P. C. (2016). Motivação, satisfação e comprometimento: Um estudo sobre o trabalho voluntário em megaeventos esportivos. Revista Economia \& Gestão, 16(42), 89-115.

Sarmento, J. P., Pinto, A., Costa, C. P. d. \& Silva, C. A. F. d. (2011). O evento desportivo como factor de desenvolvimento. Revista Intercontinental de Gestão Desportiva, 1(1), 1-14.

Shonk, D. J. \& Chelladurai, P. (2008). Service quality, satisfaction, and intent to return in event sport tourism. Journal of Sport Management, 22(5), 587-602.

Shonk, D. J., Greenwell, T. C., Bravo, G. \& Won, D. (2012). Site selection, satisfaction, and intent to return: An exploratory study of the perceptions of rights holders of small-scale sporting events. Journal of Convention \& Event Tourism, 13(1), 1-15.

Veal, A. J. \& Darcy, S. (2014). Research methods in sport studies and sport management: A practical guide. New York: Routledge.

Wicker, P., Hallmann, K. \& Zhang, J. J. (2012). What is influencing consumer expenditure and intention to revisit? An investigation of marathon events. Journal of Sport \& Tourism, 17(3), 165-182.

Wilson, R. (2006). The economic impact of local sport events: significant, limited or otherwise? A case study of four swimming events. Managing Leisure, 11(1), 57-70.

Yin, R. K. (2013). Case study research: Design and methods (5 ed.). California: Sage Publications.

Ziakas, V. \& Costa, C. A. (2011). Event portfolio and multi-purpose development: Establishing the conceptual grounds. Sport Management Review, 14(4), 409-423. 